Research paper

\title{
A rebuttal to "The proximal origin of SARS-CoV-2": \\ Covid-19 is plausibly from a manmade virus. Emergency regulatory reform for stopping epidemics
}

Timothy R. Stout timothystout@yahoo.com
This is a preprint and has not been peer reviewed. Version 6 September 3, 2020

Original publication date June 3, 2020

https://www.doi.org/10.31219/osf.io/usx58

Copyright by the author. This is an This is an Open Access article published under a Creative Commons 0 license. It may be freely copied and distributed without further permission provided credit is given to the author.

\section{Abstract}

A recent letter in Nature Medicine (www.doi.org/10.1038/s41591-020-0820-9) claims that genomic analysis of SARS-CoV-2 shows that it is clearly not manmade and that natural evolutionary causes are adequate to explain its appearance. By contrast the case is made here that genomic analysis of the virus shows that directed evolution, a human engineering process honored by the 2018 Nobel Prize in Chemistry, provides superior explanation for its origin. The simultaneous appearances of a new RBD backbone, a new polybasic cleavage sight, new fusion improvements, asymptomatic transmission, and an initial stable mutation rate indicative of mature adaptation to a host work together to lead to a common conclusion: directed evolution provides more plausible explanation of the virus's origin than does natural evolution. The major concern here is not who might have made the virus, but rather that technology already exists and is already well-understood throughout the world which is capable of creating new, future pandemics and appear to be the proximal cause of Covid-19.

A pandemic emerges because there are no effective drugs to stop it. Traditional new-drug approval laws effectively prevent newly developed drugs designed to fight a pandemic from being used soon enough to prevent it from becoming entrenched. The Stout Protocol presented here provides a plausible solution to this problem: A Procedural Specification defines the drug approval protocol and is converted into a software program which is then downloaded into a computer. No personal interactions are needed between the government approval agency and the sponsor of a new drug; the computer controls interactions. An approved drug sponsor is immediately given a limited number of volunteers for direct human testing. A physician monitors the results and reports them on a scorecard. The computer processes the scorecards and immediately computes a revised score based on the physicians' reports. The score determines how many patients a company is allowed to treat and how much monitoring for effectiveness and adverse effects is required after a drug is administered. If a score drops below a predefined value, the drug is removed from the program.

List of abbreviations used in this letter:

$\begin{array}{ll}\text { CV19 } & \text { Covid-19 } \\ \text { The Letter } & \text { The Letter in Nature Medicine posted at www.doi.org/10.1038/s41591-020-0820-9 } \\ \text { CEER } & \text { Center for Emergency Epidemic Response } \\ \text { DE } & \text { Directed evolution } \\ \text { hACE2 } & \text { human ACE2 } \\ \text { MjACE2 } & \text { Manis Javanica (a.k.a. Malayan pangolin) ACE2 }\end{array}$




$\begin{array}{ll}\text { P-CoV } & \text { pangolin coronavirus } \\ \text { PCS } & \text { polybasic cleavage site } \\ \text { RBD } & \text { Receptor-binding domain of a corona virus spike protein } \\ \text { SC1 } & \text { SARS-CoV } \\ \text { SC2 } & \text { SARS-CoV-2 } \\ \text { S1 } & \text { The S1 subunit of a coronavirus S (spike) protein } \\ \text { S2 } & \text { The S2 subunit of a coronavirus S protein }\end{array}$

Key words: man-made, bioengineered, directed evolution, backbone, polybasic cleavage, fusion, asymptomatic, pangolin, Stout Protocol, Covid-19, 10.1038/s41591-020-0820-9

\section{Introduction}

In a recent letter (The Letter) to Nature Medicine concerning the origin of SARS-CoV-2 (SC2), K.G. Andersen et al state in their opening comments, "Our analyses clearly show that SARSCoV-2 is not a laboratory construct or a purposefully manipulated virus." ${ }^{1}$ In other words, the authors claim that the evidence clearly shows that the virus is not manmade. By contrast, genomic analysis appears to be more consistent with a man-made origin using directed evolution (DE).

In the process of working through a rebuttal to The Letter, it appeared that current regulatory standards are not suitable for the rapid responses required to stop a pandemic. A suggested alternative approach was developed which is presented as the second part of this document. The two parts are presented together in an effort to show a unified picture of the how the pandemic might have begun and how it might be ended in a relatively short period of time in a single document.

\section{Part 1. Plausible Origin of SC2}

There are three issues of contention about The Letter: 1) The analyses presented in The Letter are based on obsolete technology. They do not recognize the capabilities of modern science as represented by DE, which is a standard approach commonly used today. This rebuttal will attempt to show that DE provides the best available explanation for the appearance of SC2. Thus, The Letter avoids discussion of the actual issues that should have been discussed to make their case. 2) The Letter discusses predictions made by a certain model developed by Y.Wan et al. ${ }^{2}$ However, the Wan model assumes a viral backbone similar to that of the SARS-CoV (SC1) virus that caused the SARS pandemic two decades ago. Yet, even The Letter acknowledges that the backbone of SC2 is "irrefutably different" from that of SC1. Hence, the model does not apply to SC2. Conclusions based on an irrelevant model are irrelevant, particularly for products designed by DE. 3) The assumption is made that if the virus were manmade, that "one of the several reversegenetic systems available for coronaviruses would probably have been used." This is only assumption, but it is treated as proof adequate to make the case clearly conclusive. All subsequent discussion on SC2 treats its origin as proven not to be manmade. This is poor logic at best. Moreover, when a new tool was needed to reverse engineer the newly published sequence of the SC2 genome, it was quickly designed. Within months of the release of the SC2 genome, two different, unrelated reverse-genetic systems were designed, used, and led to test results worthy of report. ${ }^{3,4}$ Reverse-genetic systems capable of transforming a computer nucleotide sequence into synthetic viruses the equivalent of SC2 are easily within the scope of tools offered by DE. When looked at carefully, not a single argument presented in The Letter appears to have merit. Invalid 
conjectures and assumptions were presented as fact. This does not represent proper, relevant, modern scientific methodology.

There are five characteristics of SC2 that distinguish it from SC1:

1) A new, never observed backbone up with up to 20 times the binding efficiency with human ACE2 (hACE20) than provided by SC1.

2) A polybasic cleavage site (PCS) insertion that allows the modified SC2 backbone to be cleaved after binding in order for fusion to proceed when the new backbone is used.

3) Membrane fusion improvements plausibly leading to increased infectivity of the virus.

4) Rapid viral growth in the upper respiratory system plausibly leading to early, "symptomless" spread of the virus before the main infection of the lower respiratory system is established.

5) Stable genome at initial appearance.

DE appears to offer a more plausible explanation for each of these than does natural evolutionary processes. For all of these new traits to make a sudden appearance simultaneously without any observed evolutionary history in a natural environment would be consistent with DE origin but difficult for natural evolutionary processes.

Despite the above problems with The Letter, which are serious from the perspective of proper scientific analysis, there is something plausibly far more serious. The Letter teaches away from the preparations needed to stop potential future pandemics once a new virus has appeared from whatever origin. The authors conclude their letter with the suggestion that more environmental studies are needed to assess the risk of future spillovers. However, this does nothing to stop an epidemic or pandemic once it is underway and diverts attention from proper current preparation required to provide improved tools and new regulatory policies to stop the current one as well as futures ones when and if they do occur. We are currently in the middle of a world-wide pandemic. As of May 29, 2020, there were 108,059 reported deaths in the USA from Covid-19. ${ }^{5}$ The world's economy is being destroyed because of it. We do not need academic discussion about how the virus originated and which spillover might have caused it. We need immediate therapeutic treatments to stop it.

The primary purpose of this rebuttal is triple fold: 1) To draw attention to the serious situation presented by modern biotechnology and the risks it presents for possible new viral outbreaks. 2) To focus attention on technological improvements needed to provide better tools for fighting future viral outbreaks. 3) To draw attention to suggested changes in regulatory laws concerning emergency approval of new treatments during a pandemic. This is the primary focus of the second part of this document. The greatest concern with The Letter is the authority with which it speaks while diverting attention from all three of these critical issues.

\section{Directed Evolution Technology}

\section{General Discussion}

Three scientists who discovered significant advances in DE technology were awarded the Nobel Prize in Chemistry in 2018-Frances H. Arnold, George P. Smith, and Sir Gregory P. Winter. This technology is directly applicable to virus bioengineering. Its principles are commonly used throughout the world in modern biochemical laboratories today. It appears that a technology significant enough to warrant a Nobel Prize and which defines modern technology that is already in widespread, general use should have been addressed in The Letter, not ignored. 
The Nobel Foundation made the following statement concerning the importance of Frances H. Arnold's work leading to her share of the 2018 Prize:

Directed evolution of enzymes and binding proteins is a man-made procedure built on molecular insights, which moves the evolution process into the laboratory and speeds it up. The procedure relies on intended variation of protein sequences at a defined level of randomness. This is coupled to engineered screening and selection strategies. Directed evolution is an iterative procedure which involves the identification of a starting state protein, diversification of its gene, an expression and screening strategy, re-diversification, re-screening, and so on until a satisfactory performance level in terms of enzymatic activity, binding affinity or specificity is reached. ${ }^{6}$

Dr. George P. Smith and Sir Gregory P. Winter, the other co-winners of the 2018 Nobel Prize in Chemistry, also helped found DE. Among the advancements they discovered leading to their portion of the prize was how to use DE to design new antibodies. They discovered the very approach currently being franticly used by many researchers at this minute in an attempt to provide new antibody treatments for SC2. It is difficult to understand how the authors of The Letter not only ignored but actually taught against something so well established and so relevant to this subject.

The Nobel Foundation mentioned that a primary characteristic of DE is its ability to provide improved performance in proteins, using new amino acid sequences not previously seen. This would be of particular value for researchers to use in developing new viruses. Perhaps the most important added tool over simple reverse engineering of currently observed constructs is the potential application of controlled, large-scale randomization to predetermined portions of a viral genome while keeping the remainder stable. A researcher is no longer limited to designing structures and functionality that he understands or that he can copy from nature. He can prescribe hundreds of millions or in some cases even hundreds of billions of random variations for a specified portion of a gene. This can be done for different portions. The results can then be recombined into a completely new virus having specifically designed traits.

The potential technique goes beyond this. With precise modeling tools available, even trillions of variations can be introduced and tested in software. Physical fabrication does not need to be performed except on a reduced number of samples.

New features requiring the simultaneous appearance of multiple, obscure mutations present an almost insurmountable problem for a process based on a succession of point mutations. There is nothing to favor selection of any given mutation potentially useful in a final assembly unless the others required also appear simultaneously. By contrast, the inherent nature of $D E$ is to provide a large number of concurrent, semi-controlled random changes in parallel across a large number of nucleotides. It is not required for a researcher to understand in advance what he will ultimately create. He can make trillions of changes to his starting material, can test them with computer simulations first and thus limit actual physical implementations to those most promising, physically test hundreds of millions of variants, and then analyze the results to determine the changes that "accidently" uncovered something useful. This process can be repeated as many times as desired, ostensibly improving on the earlier improvements. This procedure has proven so effective across so many fields of science and engineering that it led to the 2018 Nobel Prize in chemistry, shared among the three most important scientists discovering it. This is a basic approach of modern bioengineering technology.

Two good reviews of the current capabilities of DE are by Anna J. Simon et $a l^{7}$ and $\mathrm{K}$. Standage-Beier and X. Wang ${ }^{8}$. 
SC2 structural overview. A spherical lipid membrane envelope encompasses an RNA genome. Protruding from the membrane are numerous spikes. Each spike is composed of three identical copies of S (spike) proteins joined together. An S protein is composed of 2 subunits, S1 and S2, initially joined together in one continuous string. S1 finds an hACE2 receptor in a host cell using its receptor binding domain (RBD) and then binds the virus to the receptor. After binding, the S1 and S2 subunits are cloven into two separate proteins by enzymes supplied by the host. Then, after cleavage a two-step process will draw the viral and host membranes together. The S2 subunit includes three separate peptides-FP, HR1, and HR2. In the first step, the HR1 and HR2 peptides combine to form a long, six-helix bundle $(6 \mathrm{HB})$. One end of the bundle attaches to the virus membrane. The other attaches to the base of the FP. During this step, the FP is projected towards, pierces, and fuses to the host membrane. When the fusion is complete, the 6HB undergoes a configuration change which draws the FP towards the virus membrane, allowing the host and virus membranes to draw close enough to fuse together ${ }^{9}$. In the case of SC2, actual entry into the host is by a process called encapsulation ${ }^{10}$. When a spike binds to a host cell, then neighboring spikes proceed also to bind in a growing pattern. Because of innate coronavirus spherical structure, this process ultimately results in the virus becoming completely engulfed within the host membrane. The ACE2 receptors enter the cell along with the virus. Once the virus-hACE2-membrame complex forms a complete sphere, it is released within the cell, the components disassociate and the virus sets up manufacturing of new virions. ${ }^{11}$

1. Backbone. The Letter mentioned that the genetic data irrefutably show that SARS-CoV-2 is not derived from any previously used virus backbone. The backbone is a part of the spike protein that fits over a convex bulge on the surface of the hACE2 receptor. Two flexible peptide loops are also attached to the backbone. Various amino acids present in the backbone and in the loops bond with nearby amino acids in the receptor, forming salt bonds, hydrogens bonds, and Van Der Waals Force bonds. In coronaviruses, the affinity between viral RBD and host receptor determines which host is susceptible to infection as well as its level of susceptibility.

T. Hien et al simulated in GROMAC software the differences in bonding between SARS-CoV and SARS_CoV-2 viruses with the hACE2 receptor. They state,

Our results show major mutations in the 2019-nCoV RBD with respect to the SARS-CoV RBD occurring at the interface of RBD-hACE2 complex. These mutations make the 2019-nCoV RBD protein backbone much more flexible, hydrophobic interactions are reduced, and additional polar/charged residues appear at the interface. We observe that higher flexibility of the 2019-nCoV RBD with respect to the SARS-CoV RBD leads to a bigger binding interface between the 2019-nCoV RBD and hACE2 and to about 20\% more contacts between them in comparison with SARS-CoV. Taken together, the 2019-nCoV RBD shows more stable binding interface and higher binding affinity for the hACE2 receptor. The mutations not only stabilize the binding interface, they also lead to overall more stable 2019-nCoV RBD protein structure, even far from the binding interface....

Despite very similar sequence identities, four important mutations in the new viruses play important role in this stronger binding process, namely $Y$ to L455, VP to El471-472, -PP toGVE482-484, and Y to Q498 (the indices are that of SARS-CoV-2 virus). These mutations all lead to a reduced internal rigidity of the viral protein backbone near its binding interface and add polar and charged residues to the interface. The higher flexibility in the backbone of the new viruses then allows it to move closer to the human receptor protein surface, and to bind stronger to the receptor using non-bonded interactions. Overall, the 
coordination number between the proteins increases by $20 \%$ in the cases of the new SARS$\mathrm{CoV}-2$ viruses. This increase in different non-bonded interactions (electrostatic, van derWaals, hydrophobic) overcome the loss of one hydrogen bond between the proteins, leading to a stronger binding free energy....

Preliminary estimate of the binding free energy agrees reasonably with the experimental data that SARS-CoV-2 viruses show 10 to 20 folds higher binding affinity for ACE2 receptor as compared to SARS-CoV virus. ${ }^{12}$

These changes result in different bonding interactions between the viral RBD and the hACE2 receptor. They also result a different electrostatic surface potential mapping of the SC2 backbone. ${ }^{13}$ Perhaps the electrostatic mapping change partially explains the necessity of the polybasic cleavage site discussed next.

A 10 to 20 -fold increase in bonding affinity would plausibly result in intensive selection pressure to promote the appearance of the new backbone. Yet, despite its plausible improvements and the lengthy time lineage coronaviruses have existed, SC2 represents the first time it has been observed. This suggests that there is not a clear step-by-step path from the SC1 backbone to SC2's, but that many of the changes might need to occur in parallel before any have value. Of possible critical significance is the insertion of glycine (G) at amino acid position 482 mentioned above. This specific insertion is plausibly a rare event. If it is deleterious in itself but favorable on simultaneous appearance some of the other listed mutations, it could be quite difficult to appear naturally. Since a large number of parallel mutations is characteristic of DE and difficult for natural mutation, it appears that $D E$ is the more plausible source of the new backbone.

2. Cleavage-The SC2 virus is characterized by insertion of a polybasic cleavage site (PCS) where the subunits $\mathrm{S} 1$ and $\mathrm{S} 2$ of the $\mathrm{S}$ protein are cloven and become two separate proteins as needed for fusion to begin. Inclusion of A PCS is unique for Corona viruses related to SARS. It has never been observed in any closely related coronavirus, although it is found in more distantly related ones. However, the specific nucleotide sequence of PRRA|RS used in the cleavage site appears to be unique, never having been observed anywhere else. At this point it is unknown if this particular sequence is associated with any unique behaviors.

Markus Hoffmann et $a l^{14}$ reported on experiments showing that if the SC2 PCS is removed from a proteome, SC2 no longer has the ability to infect. I suggest that this might be because of the change in static field characteristic of the new backbone, which was discussed above. If this is the case, then the SC2 backbone could not have evolved naturally without a preexisting PCS. It would be within normally anticipated capabilities for DE to provide both a never-before-seen PCS and a never-before-seen RBD backbone in a simultaneous, interacting appearance. By contrast, it appears less likely for natural processes to do this.

3. Membrane Fusion. As a general observation the domains of the $\mathrm{S} 2$ subunit are stable and undergo minimal mutation. This suggests that they might already be close to optimal performance. Nonetheless, S. Xia et al reported,

SARS-CoV-2 showed a superior plasma membrane fusion capacity compared to that of SARS-CoV. We solved the X-ray crystal structure of six-helical bundle (6-HB) core of the HR1 and HR2 domains in the SARS-CoV-2 S protein S2 subunit, revealing that several mutated amino acid residues in the HR1 domain may be associated with enhanced interactions with the HR2 domain.... ${ }^{15}$ 
There are two statements of significance here. The first is superior fusion capacity of SC2 over SC1. The second is their discovery mentioned in the text of eight specific mutations that plausibly lead to this increased performance. They summarize this analysis with the suggestion this could lead to "increased infectivity of the virus":

These results suggested that the multiple replacements in the HR1 domain of emerging SARS-CoV-2 virus could enhance the interactions between HR1 and HR2 domain to further stabilize the 6-HB structure, which may lead to increased infectivity of the virus.

The appearance of eight new mutations leading to observably superior performance in already highly stable, conserved domain is unusual enough. To have so many appear in SC2 simultaneously with the appearance of anew backbone and a new polybasic cleavage site plausibly favors appearance by DE over natural evolution.

4. Aysmptomatic Transmission. A unique characteristic distinguishing Covid-19 from SARS is asymptomatic transmission in the early days of an infection. An early preprint article discusses a plausible reason for this. ${ }^{16}$ The evidence is based on in vitro analysis:

The upper respiratory tract (nose, sinuses, pharynx, or larynx) tends to be about four degrees Celsius lower in temperature than the lower tract (bronchial tubes and lungs). Incoming ambient airflow cools the upper portion of the respiratory system more than the lower. The authors report that host temperatures play a critical role in influencing the replication rates of a wide range of coronaviruses as well as the "dynamics of host immune response." In vitro investigation by the authors showed that for the first 48 hours after infection at 33 degrees Celsius - representative of upper respiratory tract temperatures-SC2 was observed to replicate about one hundred times as rapidly as SC1. However, at 37 degrees Celsius - a higher temperature which is more representative of lower respiratory tract temperatures - the replication rates between SC1 and SC2 were the same. This suggests that the SC2 virus could be expelled through coughing, sneezing, wiping the face with hands, etc. before lower respiratory symptoms developed, resulting in asymptomatic transmission. In conclusion the authors suggest that the observed increase in replication rates between SC2 and SC1 in upper respiratory system tissue is because of mutations in the genetic code at currently unknown locations.

Technically, the "asymptomatic transmission" is plausibly due to upper respiratory tract infection instead of the lower respiratory tract causing the severe problems. There may be minor symptoms associated with this infection, but they are not those typically associated with Covid-19 and frequently can be mild enough to be barely noticeable.

5. Stable genome at initial appearance. When a person solves a jig-saw puzzle using just the pieces and without a picture to work with, he does not know what the final picture will look like until the last piece fits, even though the picture gradually reveals more as more pieces are added to the ones in place. We will now consider a final piece in this analysis that ties all of the others together. It also greatly strengthens the case for SC2 being a product of DE, not natural evolution.

On May 1, a little over three months from when Covid-19 began its spread, S. Zhan et al submitted a preprint titled SARS-CoV-2 is well adapted for humans.... ${ }^{17}$ They noticed that when a viral spillover occurs and leads into an epidemic, there is typically an initial rapid mutation rate as the virus adapts to various unique characteristics of the new host. By contrast, SC2 appears to start off well adapted for human infection from the time of its first appearance: "It appears genetically stable and not under much pressure to adapt." They performed a side-by-side comparison of evolutionary dynamics between SC1 and SC2 in three-month intervals, starting with the initial appearance of each. When SC1 first appeared about two decades ago, it's genome 
rapidly adapted in response to selective pressure. This is a natural characteristic of spillovers. As the virus mutated under the influence of selective pressures, it gradually became better adapted to its new host, the selective pressures were reduced, and the viral genome became more stable.

DE allows focus on improvements of selected features while keeping everything else fixed. For instance, it would be possible to focus on each of the above-discussed features separately, combine the results in their improved forms, and repeat the process as yet new finds are uncovered. As each viral feature is processed for optimum adaptation for a human host, it becomes more difficult for natural processes to improve on it. Then, when all of the features are assembled into a new virus and it is introduced into a population, it could already be at nearoptimal adaptation. Its genome would be relatively stable, already being well adapted to its target host. This is precisely what was observed with SC2. By contrast, SC1 did not go through preoptimization before entering public infectivity. Initially, there were many, frequent adaptations. Only later in its life cycle did it develop a stable genome. This is perhaps one the strongest evidences in support of a DE origin for SC2. It appears implausible that natural processes could have provided the adaptions observed in its back bone, PCS, fusion components, and extensive pre-symptomatic spread with sudden, simultaneous first appearance in already welladapted form. By contrast, this would be anticipated with a DE origin.

Pangolin origin of the backbone? The Letter discusses the possibility that SC2 might be the result of a spillover from a virus infecting Malayan pangolins, which they refer to as pangolin coronavirus (P-CoV). Discussion in The Letter illustrates just how close the relationship is between P-Cov and SC2, giving a detailed graphic proteome analysis between them. A common perspective among many scientists today is that the pangolin backbone had a recombination spillover into SC2 but through an as yet unknown intermediary host. However, careful examination reveals some unexpected issues which may challenge this perspective.

On January 10, 2020 a team of scientists at the Wuhan Institute of Virology published the SC2 genome online at Genbank ${ }^{18}$. The Chinese government became angry that this information was leaked. Ten days later, the genome of a corona virus which infects Malayan pangolins by means of the Malayan Pangolin ACE2 receptor (MjACE2) was uploaded to the NIH website. ${ }^{19} \mathrm{~A}$ month later the same team uploaded the genome to Genbank ${ }^{20}$ along with dialogue about how PCoV was taken from three sick pangolins that were being smuggled into China and captured by customs authorities. ${ }^{21}$ Remarkably, P-CoV had almost exactly the same RBD as SC2. Plausibly, Covid-19 was the result of a spillover from the nearby wet market from an infected pangolin. The uploaded sequences provided data used in the genomic analyses of The Letter.

For a number of reasons, I wonder about the legitimacy of the pangolin genome as posted. Is it possible the posted genome exists only as a computer construct and not in pangolins? There are four concerns discussed here that are consistent with this perspective. They can potentially be resolved by proper computer modeling.

1. The MjACE2 receptor is different in structure and functionality than hACE2. For instance, in general there are 20 key amino acids in mammalian ACE2 structure which viruses use for bonding; these amino acids vary between different hosts species. Only 14 of these 20 amino acids are similar between MjACE2 and hACE2. ${ }^{22}$ This much discrepancy leads to a serious question. Can the reported pangolin RBD sequence even bond to MjACE2? It is plausible that it might not or only do so weakly. This has a direct bearing on the legitimacy of the published MjACE2 genome. It is possible the authors were unaware of the differences between MjACE2 and hACE2 when the genomes and proteome were uploaded and had not anticipated this problem. 
This question may plausibly be resolved by using modeling software such as GROMAC ${ }^{23}$. The GROMAC model used by Tien et al as discussed above regarding the backbone could be very suitable for this purpose if the MjACE2 genome were to be substituted for that of hACE2.

2. The new SC2 RBD backbone binds to hACE2 up to 20 times as efficiently as the SC1 backbone. Even if the reported pangolin RBD does bond to its receptor, there is still a serious question to be answered: How is it that an animal with such different ACE2 receptor bonding characteristics could be the source of a new, hitherto unseen backbone which bonds so effectively to human receptor-up to 20 times more efficiently than anything observed previously?

3. The pangolin proteome does not have a PCS, as even The Letter acknowledges. Earlier it was shown that when humans implement the SC2 backbone, S1/S2 cleavage is inhibited unless a PCS is provided. Without cleavage no fusion takes place and the host is not infected. It was suggested that cleavage failure is due to the changed electric charge field of the new SC2 backbone interfering with the traditional cleavage process. The pangolin proteome has no PCS but reputedly uses the new backbone. One would anticipate that the same issues that prevent SC2hACE2 cleavage and fusion without a PCS would have the same effect in pangolins. It could be profitable to model this relationship also.

4. If the SC2 backbone is the product of DE, which is the proposed understanding of this rebuttal, it is plausible that the new backbone was the final product of a series of experimental runs having billions of random, new combinatory sequences representing parallel, simultaneous mutations in the RBD-bonding amino acids. Historically, coronaviruses have been around a long time infecting ACE2 receptors across a wide range of animals. The SC2 backbone has never been seen until now and reputedly also in P-CoV. It would be interesting to model a Bat RaTG13 RBD with its traditional backbone and determine what kinds of mutations would be required to provide a path giving selective advantage of steps leading to the appearance of the new backbone used in SC2. According to the principles of evolutionary theory, many small steps are more plausible than a few large ones. The more parallelism required to make the transition, the stronger the evidence becomes suggesting DE as the originating cause of SC2.

Thus, there are four issues that make the posted $\mathrm{P}-\mathrm{CoV}$ genome a paradox. If properly constructed models were to show that despite these concerns, P-CoV to MjACE2 bonding is not only effective, but also allows S1/S2 cleavage and does not interfere with fusion, then that not only would confirm the legitimacy of the posted genome, but also could lead to a better understanding of how the spillover could have occurred.

Conclusion of the technical analysis. The Letter began with the assertion, "Our analyses clearly show that SARS-CoV-2 is not a laboratory construct or a purposefully manipulated virus." This is a very strong statement for a scientific paper, where statements are typically qualified by words such as "plausible" or "appears." Yet, 1) The logic used to justify such a strong conclusion was poor. 2) The arguments were straw-man, not based on the capabilities of current technology. 3) The initial appearance of SC2 was already extremely well-adapted for humans with little room for natural improvement, which is consistent with DE origin and not natural origin. The combined significance of all of these suggests high plausibility that SC2 is a laboratory construct using DE. Yet, as serious as these problems may be concerning the assertions presented in the letter, to me personally they are not as serious as the following issues.

\section{Final remarks - What next?}

The above discussion was intended to promote general awareness of just how dangerous modern technology has already become. Man has now acquired the technology to create new 
viruses capable of causing a pandemic and has plausibly done so. Deliberate or accidental release is irrelevant. The intent is to show how serious a threat modern biotechnology has already become to the survival of mankind and how widespread the knowledge to implement this technology already is. There is no turning back. Ignoring or covering over the issue diverts attention away from developing the best possible defenses against new viruses that could arrive. Well-established advances in science in use throughout the entire world and which have resulted in a Nobel Prize cannot be unlearned by society as a whole. The world is in no position to deal with a series of pandemics such as Covid-19.

Three remaining concerns are suggestions on how best to focus limited resources in order for them to be most productive.

The first of these is to suggest proper areas of focus on how best to fight an ongoing pandemic, such as developing more efficient therapeutics or repurposing existing drugs. The second concern is to develop a more rapid means to go into mass production for approved therapeutics. The third is for immediate regulatory changes to allow rapid implementation of critically needed technologies as they become available. These three areas of focus are plausibly the most critical need for the survival of mankind. The most serious defect of The Letter is its diversion from these issues into environmental studies. For instance, we are currently in a pandemic. Does it really matter to an infected person dying in a hospital whether he is sick from a zoonotic spillover or an accidental release of a man-made virus? He needs immediate, effective treatment, not academic discussion.

Goals for rapid deployment. The following are subjective goals for technological advances which could plausibly stop a potential epidemic before it becomes widely established. These are items for discussion and goal setting and not based on any particular advancements or technology. An arbitrary goal of two weeks is proposed for the time from when a virus is isolated for genome sequencing until therapeutic new drugs are available for use under controlled conitions in fighting a pandemic. The suggestion is that research funds be focused on meeting these goals, not environmental studies trying to guess where a zoonotic spillover might originate:

1) 5 days. Genomic identification and comparative analysis with existing genomes and public disclosure. The goal is that within 24 hours of when a new virus capable of causing an epidemic is isolated, its genome can be sequenced, an initial analysis of its similarity to other viruses with genomes stored in Genbank, posting of the information on a governmental website, and public announcements of the posting sent to news media and to those who have signed up on an e-mail list to receive such announcements, to the email list.

2) 15 days. Development of new therapeutic treatments designed to stop the infection cycle of an epidemic or pandemic. Therapeutic measures are needed once an epidemic has become established. Typical therapeutic measures would be antibody treatments and relabeling treatments approved for other conditions. One company has already done this in about 60 days, as will be discussed later. The target is for a six-fold speed up. Therapeutic treatments target those who are already infected. These people need immediate treatment.

3) 10 days. Time to manufacture newly designed drugs of sufficient purity to start limited trials on already-infected people. Manufacturing techniques to allow subsequent extremely rapid ramp up in manufacturing capacity as the confidence level in the new drug is established.

Under this schedule, it would take one month to progress from isolation of a new virus until new therapeutic treatments become available to treat it and permission is given to start human trials under the Stout Protocol. 
Covid-19 is now the third leading cause of death in the United States. The nation's economy is in shambles. People cannot mix freely in private or public settings. Yet, looking back it appears that these problems are needless. By now, the pandemic should be over or at least be well under control. New drugs designed to fight it were announced five months ago. Yet, they still sit on a shelf because of traditional new-drug approval laws which are extremely time-consuming in their application. They are not appropriate for the emergency conditions of a pandemic. In this document, the Stout Protocol is presented as a solution to fix this problem. Getting this protocol approved is one of the most urgent needs facing our country.

The Stout Protocol fixes this problem by using a computer to control the new-drug approval process, eliminating personal interactions between a governmental bureaucracy and a drug sponsor. The agency still controls the process, but indirectly through the computer. Computer interactions are instantaneous. This is the kind of response needed during a pandemic.

The following diagram shows the various components used in the protocol and how they relate to each other:

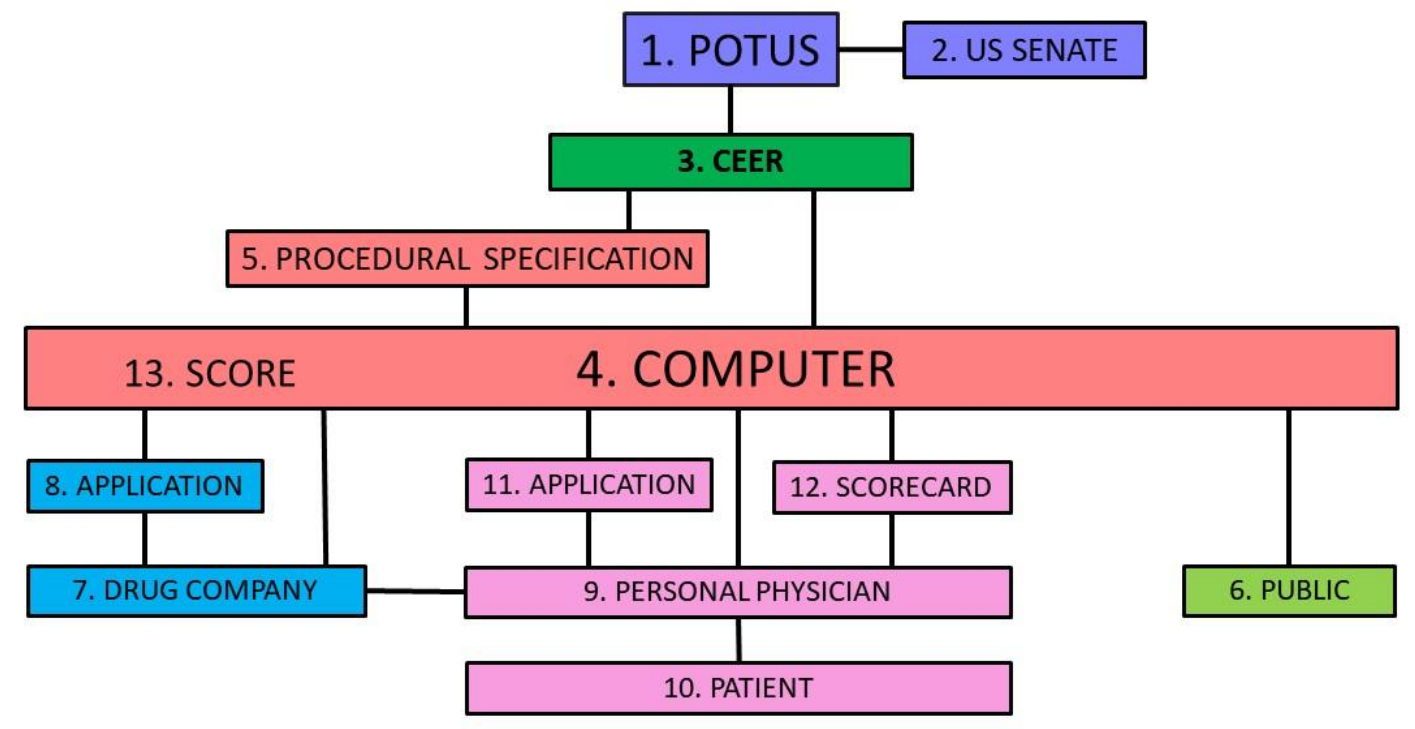

The Stout Protocol

Fig 1. Diagram of protocol components and their relationships as applied to the United States

\section{Description and Function of Diagram Components}

1. President of the United States (POTUS). In the United States, POTUS has direct executive authority over administration of the protocol, similar as he is commander-in-chief of the US military for emergency threats from enemy militaries. When an epidemic or pandemic appears to be of 
sufficient threat POTUS declares a Resolution of Epidemic Emergency. The disease causing the emergency is defined. Other countries could follow a similar structure.

2. United States Senate. The Resolution of Epidemic Emergency is sent to the United States Senate for ratification. Upon ratification by the US Senate, the Stout Protocol officially goes into effect. When the sponsor of a new drug that falls within the scope of the epidemic applies to the government, it is subject to the new laws instead of the standard ones. Senate confirmation is required because of the legal significance of removing drug approval from traditional channels into the new one described here. The protocol is effective for a predetermined length of time, such as two years. The time may be extended by future resolutions and confirmations. This is an emergency protocol for use under emergency conditions only.

3. Center for Epidemic Response. Administrative authority for implementing the protocol is placed in a governmental agency referred to here as the Center for Emergency Epidemic Response (CEER). CEER may be a stand-alone agency independent of the FDA or be under its jurisdiction.

The focus of CEER is to fight the epidemic as effectively and rapidly as possible while still maintaining reasonable standards of safety.

4. Computer. CEER exercises indirect control over the drug approval process using a computer. The computer represents a single point of contact between the government (CEER) and the Public. It provides a general information resource about the pandemic. It provides detailed information about testing requirements under the protocol. It tracks the status of all drugs in the protocol and makes the information public. It immediately analyzes status reports of physicians monitoring the results of drug treatments. Physicians' reports are the basis for determining in real-time the degree of expansion in testing allowed for a new drug or conversely its elimination from the program. The reports over a period of time also determine the scope of monitoring required after treatments with the drug.

Currently, the interaction between the FDA and a drug sponsor is extremely time consuming. It ensures a pandemic is allowed to run its course-approval of new drugs designed to fight it are tied to a slow, time consuming protocol not compatible with the urgency presented by a pandemic. An example of this is the current situation where Covid-19 is the third leading cause of death in the United States at this time, while new drugs capable of fighting it sit idly on a shelf. In the new protocol this entire delay is removed by the real-time processing capabilities of the computer. Any imperfections of this approach are seen as insignificant compared to the consequences of allowing a pandemic to run its natural course unimpeded while drugs that might be useful to fight it are by law unable to be tested or used.

5. Procedural Specification. The Procedural Specification defines the approval/control process. Since every pandemic is unique in its characteristics, CEER is given much latitude in the protocol definition. It is a "living document." As more is learned about the pandemic and the pathogens causing it and about the results of various treatment approaches, the Procedural Specification is modified. Also, as the number of people infected becomes larger, the more liberal the increases in numbers of patients can be. The responsibility of CEER is to monitor all aspects of the pandemic and results of treatments administered to patients and then to control the approval process by making changes to the procedural specification as appropriate. 
The specification is coded into computer software which is then loaded into the computer. Patient monitoring reports are in numerical format for simple computer analysis.

This approach is suitable for a pandemic where all drug companies are focused on a single pathogen under emergency conditions. It is not suitable for the normal drug approval scenario, where the conditions and circumstances related to each new drug application are unique and need to be analyzed on an individual basis.

6. Public. The Public represents everyone outside of CEER who interacts with the computer. It includes the public at large, people who might be interested in participation in new-drug trials, those are who taking part in new-drug trials, drug companies, physicians of patients under treatment, and conceptually even other governmental agencies doing their own research or other interactions. In general, everything possible about the trials is made public. For security purposes, certain aspects may be need to be kept confidential, but the general philosophy is to keep confidentiality at the minimum possible and only when specific reasons justify it.

Public awareness serves two purposes. First, patient participation in the approval program is voluntary. The drugs being tested have not been formally approved and the patient has the right to know what he is volunteering for. Furthermore, if a drug sponsor knows the results of its treatments are going to be immediately and irrevocably announced publicly, this serves as a control for it to be careful and not over-zealous in using large doses too quickly. Bad results in the first few patients could effectively discourage any future volunteers from participation as well as the drug's quick removal from the protocol. In effect, the drug sponsor becomes accountable to the public instead of a bureaucratic agent. In a pandemic, this is viewed as the most effective way to protect the public. During a pandemic the pressure of urgency results in elimination of many of the normal procedural controls. A drug sponsor gives up confidentiality for the privilege of participation in the abbreviated pandemic emergency protocol.

7. Drug Sponsor. A drug sponsor is a drug company, university, research organization, or other governmental agency which has developed a new drug for which it desires approval for use in fighting the pandemic. All the information known about the pandemic and the pathogen causing it and which is suitable for public disclosure is posted on a public website on the computer. In some cases involving biowarfare, information may need to be kept confidential. However, this is expected not to be a normal situation.

If a drug sponsor decides to develop a new drug to fight the pandemic based on the information available, both from the CEER computer's website and other sources and if it desires to operate under the CEER protocol instead of the standard FDA protocol, then it makes an application to the CEER. If it prefers to get approval through the traditional FDA route, it makes application to the FDA. A company even has the option to do both routes simultaneously if it chooses to do so.

8. Drug Sponsor Application. CEER has authority to establish standards for sponsor participation in the program. This may vary from pandemic to pandemic. It can change within a pandemic according to the needs of the pandemic, particularly as more is learned about it and the pathogens causing it. There are two conflicting issues here. One is to ensure that a company is qualified to develop and test the drugs for which it seeks approval before it is allowed to treat volunteers. The other is to eliminate any kind of bureaucratic delay for drug test authorization when a pandemic is in progress. 
One possibility of resolving this conflict as expeditiously as possible is to have two applications. The first is sponsor qualification standards. Issues such as financial stability, corporate new-drug approval-process experience, specific fields of interest and research experience (particularly if earlier forms of new drugs have previously been taken through the approval process), and qualifications of key project personnel can be a part of this form. These standards could plausibly be pandemic independent. In this case, a list of companies meeting basic standards as qualified sponsors are kept on file. This allows CEER time to do independent confirmation of an applicant's qualifications. It is in the best interests of a potential sponsor, CEER, and the public for sponsor qualification be recognized before a pandemic sets in whenever possible.

The second application would be for authorization to use a specific drug in human trials. The new-drug application would include anything deemed relevant by CEER. Only qualified sponsors would be permitted to submit new-drug applications. The goal would be to have a set of questions that can be checked off as having been met, submitted in a form suitable for computer analysis and thus eliminating human interaction. Stringent legal penalties are imposed on fraudulent responses, particularly if volunteer patients are harmed as a result. When a qualified company represents on the new-drug application that it has met the basic standards for drug approval per the Procedural Specification, it is automatically allowed a specified number of patients for use in trials. Here is where public disclosure is important. A company is constrained to be careful not to be overzealous in trying to advance too quickly lest early bad reports destroy interest in the drug by potential volunteers. The application would also include information relevant to be posted as public information concerning the drug, how it is hoped to be useful in fighting the pandemic, perceived risks, and what the qualifications/characteristics it is looking for to participate in the trials. The qualifications criteria can be updated as appropriate in the course of the trials.

When a sponsor submits the application, the information relevant to volunteer participants in the trials is immediately posted on the CEER website.

9. Personal Physician and 10. Patient. When the Stout Protocol is in effect during a pandemic emergency, there will generally be people from the public interested in serving as volunteers for testing of the new drugs. Generally, a pandemic/epidemic exists because there are no drugs to treat it. The CEER computer provides information regarding companies and what they offer for new drugs for use in fighting it. The computer posts this information plus the criteria as determined by the Sponsor for those wanting to participate in a trial.

In general, in the interests of time, CEER will give a sponsor much latitude in determining the standards for volunteers at any particular stage of development as well as the treatment protocol (dosage per treatment, number of treatments, time between treatments, etc.) The Sponsor will be motivated to advance to safe treatment standards as rapidly as possible, all the while remembering that everything it does and their results will be closely monitored by the public. In the interests of fighting the pandemic, this seems to make more sense than a tightly controlled, prescribed pattern determined by one-on-one negotiations between a governmental bureaucrat and a drug sponsor, which are extremely time consuming. The patient and the doctor discuss between themselves whether the new drug seems appropriate or not.

11. New Patient Application. When a patient and physician agree together to make the patient available for trials, the physician submits an application providing the requested information for 
any specific drugs of interest. More than one drug may be volunteered for, but only one may be used for treatment. Up until a patient has received the first treatment, he has the option to change or decline drugs at will.

The computer forwards patient information to the appropriate drug sponsor. A sponsor will have permission to treat a specific number of volunteers at any given time. It may choose these from among those available whichever ones it judges to be most useful in helping it to evaluate a drug's behavior and effectiveness under various conditions.

12. Scorecard. The heart of the protocol is a scorecard. After a physician treats his patient with the drug, he monitors the results and records them on a scorecard, which in turn is uploaded to the computer. These physician evaluations determine how a drug progresses through the trials. $A$ scorecard also has space for verbal comments by physicians. These comments do not impact the score. Their purpose is to make CEER aware of issues that can be relevant. This can lead to modifications in the Procedural Specification if appropriate.

The scorecard lists a number of issues including the patients age, height, weight, sex, vital signs, health, the apparent effectiveness of the drug, any observed side effects, and whether the physician would recommend stopping the trial, continuing it on a limited basis, or increasing the number of people included in the trial. CEER will typically have a number of other issues to be monitored and reported as well.

Once the physician submits the scorecard to the computer, everything becomes automatic. Scorecards minus personal identification information are immediately posted as public information. The computer analyzes all of the scorecards for a given drug. The computer calculates a new score immediately upon receiving a new scorecard from a patient's doctor.

CEER has the responsibility to monitor how well the computer evaluates scorecard response and as appropriate can modify the scorecard format and/or the computer's interpretation of the data. Indirect control allows the protocol to operate reasonably effectively, but without any delay time for personal interaction between CEER and drug sponsors. The safe elimination of this delay is the primary objective of the new protocol.

13. Score. The Procedural Specification defines how to calculate a score from the scorecards. New calculations are typically performed immediately each time a new scorecard is received for a drug. As the score for a drug gets higher, additional patients are allowed into the program and gradually less monitoring for adverse reactions is required. If the score goes below a certain value, the drug is removed from the program. The new scores and scorecards are also posted immediately for public view. This is for the benefit of volunteers making final decisions for treatment.

The procedural specification is a living document. CEER continually needs to update the Procedural Specification as the pandemic changes in scope and as doctors discover newly observed issues that need to be monitored and reported.

A long-term goal is to allow eventual formal approval of drugs demonstrated to be effective and safe. They would continue to be available for treatment of the disease even after the pandemic has ended. This would be the equivalent of approval as that of a drug that has gone through the traditional FDA protocol. CEER defines the steps to this.

\section{Repurposed Drugs}

Repurposed drugs are ones that have been approved for treating certain diseases but not the ones desired. 
There can be a gray area concerning when they are allowed and when they are not. There can be a gray area of legal liability for a doctor or hospital using them this way. There can be a gray area whether insurance covers their costs. A major responsibility of CEER would be to define a protocol formally allowing a controlled use of repurposed drugs within the protocol.

A typical example of repurposing would be the Math+ protocol developed by a group of doctors treating Covid-19 first hand. It is discussed at https://covid19criticalcare.com . They found that their Covid-19 patients appeared to die from blood coagulation problems more than lung problems. They devised a treatment based on treating these problems and found they had a very high rate of success - particularly for hospitalized patients who started treatment soon enough. Yet, very few people know of this treatment and use it. The new protocol could help in these ways:

1. Provide a common site where all drugs under investigation can be easily discovered and their effectiveness tracked. This would help people find out about them and decide whether or not to volunteer to use them.

2. Provide a legal safe haven for doctors, hospitals, and other medical practitioners who might otherwise be reluctant to take the legal risk for a repurposed drug unless it is covered in the protocol.

3. Legally allow the full cost of treatments to be covered by insurance, for both hospitals and doctors.

Math+ is not the only repurposed drug with potential. It is only representative. However, if these new policies had already been in place to be allowing early-on, official testing and reporting of repurposed drugs, there is good possibility that the pandemic and its effects would not have been as severe as what are now facing.

\section{Miscellaneous Concluding Remarks}

Animal Trials. During an active pandemic, the delays required to perform animal trials are not justifiable in light of the damage the pandemic cause during the delays. Animal trials are optional unless a drug sponsor wants to perform them.

Legal protection. Once a new-drug has been registered with and approved by CEER, the company is exempt from FDA requirements for new drug approval. The drug sponsor, hospital, physician, nurse, or anyone working within the guidelines of these exemptions and using reasonable care in the exercise of their duties shall not be liable for damages due to complications from participation in the trials. Gross malpractice is not exempt. To qualify for participation in a trial, a patient and his doctor need to affirm that they understand the risks of unapproved drugs and voluntarily accept treatment with them for whatever personal benefit and reasons they might have for desiring to be included in the trials.

Conclusion of CEER's authority. Once an active declaration of emergency has been officially announced as over, the trial period is over. Continued use of unapproved drugs is not allowed. In the event of a reemergence of the pandemic, only drugs having formal approval may be used for 
treatment. CEER has the authority to formally approve drugs for use after the pandemic ends for pathogens causing the pandemic.

Normally, CEER's jurisdiction in a pandemic will continue until the resolution authorizing its active status expires without renewal.

Insurance. Insurance companies are to be required by law required to cover treatments under this set of regulations as the equivalent of an FDA approved drug. A person requiring hospitalization will be covered the same as if he were receiving FDA-approved care. A person suffering adverse effects from a drug administered in this section, such as but not limited to allergic reactions, shall receive the same benefits as if the reactions came from a drug that had already been approved. It is expected that in the overall picture, the benefit to an insurance company for this kind of support in stopping a pandemic or reducing its impact will be of greater financial benefit than allowing an epidemic to progress without control. It is to the insurance company's advantage to help fight the pandemic, even if a few early cases are problematic. It is in the public interest to ensure that patients being treated are covered by their insurance. The cost of drugs administered during treatments can be reimbursed up to a value determined by CEER, not to exceed the general cost of equivalent drugs on the market. Excluded from reimbursement are extra tests, extra physician monitoring and evaluations, and scorecard reports required as required by the Procedural Specification. These are to be born by the drug sponsor. CEER documentation is to clearly state what is to be covered by insurance; all other costs are to be born by the drug sponsor.

Therapeutic Drugs and Vaccines. The Stout Protocol is intended for therapeutic drugs, which are typically given to people already infected or at high risk of infection. The consequences from a spreading pandemic are anticipated to overshadow greatly any decline in safety as result of following the Stout Protocol. By contrast, vaccines are normally given to a large fraction of $s$ population of healthy people to allow their bodies to develop immunity. Shortcuts in the drug approval process in this case could lead to results as bad as over even surpassing those of the pandemic itself.

However, if a Procedural Specification can be designed and implemented for safe vaccine inclusion into this protocol, this can be accomplished within the structure already proposed: POTUS makes specific inclusion of vaccine treatments to be within the scope of the resolution defining the pandemic emergency. Upon Senate ratification, vaccines are then included within the scope of new drugs included within CEER's jurisdiction. By contrast, inclusion of therapeutic drugs is automatically included in a resolution. It is recommended that POTUS not recommend vaccine inclusion until CEER has a satisfactory Procedural Specification for vaccines defined and a debugged version of software to implement it functioning properly in the Computer. 


\section{References:}

\footnotetext{
${ }^{1}$ Andersen, K.G., Rambaut, A., Lipkin, W.I. et al. The proximal origin of SARS-CoV-2. Nat Med 26, 450-452 (2020). https://doi.org/10.1038/s41591-020-0820-9

${ }^{2}$ Wan Y, Shang J, Graham R, Baric RS, Li F. 2020. Receptor recognition by the novel coronavirus from Wuhan: an analysis based on decade-long structural studies of SARS coronavirus. J Virol 94:e00127-20. https://doi.org/10.1128/JVI.00127-20

${ }^{3}$ Xie et al., 2020, Cell Host \& Microbe 27, 1-8 May 13, 2020 a 2020 Elsevier Inc. https://doi.org/10.1016/i.chom.2020.04.004

${ }^{4} \mathrm{~T}$. Thao et al, Feb. 21, 2020. Rapid reconstruction of SARS-CoV-2 using a synthetic genomics platform. bioRxiv preprint doi: https://doi.org/10.1101/2020.02.21.959817

5 https://www.worldometers.info/coronavirus/country/us/ retrieved May 30, 2020

${ }^{6}$ https://www.nobelprize.org/uploads/2018/10/advanced-chemistryprize-2018.pdf

${ }^{7}$ Anna J. Simon, Simon d'Oelsnitz, and Andrew D. Ellington, Nature Biotechnology | VOL 37730 | JULY 2019 | $730-743$ | www.doi.org/10.1038/s41587-019-0157-4

${ }^{8}$ Kylie Standage-Beier1, Xiao Wang. September 27, 2016. Front. Chem. Sci. Eng. www.doi.org/10.1007/s11705-0171618-2

9 Sandrine Belouzard, Jean K. Millet, Beth N. Licitra and Gary R. Whittaker. Mechanisms of Coronavirus Cell Entry Mediated by the Viral Spike Protein. Viruses 2012, 4, 1011-1033; www.doi.org/10.3390/v4061011

10 Shuai Xia, Yun Zhu, Meiqin Liu, Qiaoshuai Lan, Wei Xu,..., and Lu Lu. 3 February 2020. Nature Cellular and Molecular Immunology. www.doi.org/10.1038/s41423-020-0374-2

${ }^{11}$ Sandrine Belouzard, Jean K. Millet, Beth N. Licitra and Gary R. Whittaker. Mechanisms of Coronavirus Cell Entry Mediated by the Viral Spike Protein. Viruses 2012, 4, 1011-1033; www.doi.org/10.3390/v4061011

12 Hien T. T. Lai, Ly H. Nguyen, Agata Kranjc, Toan T. Nguyen, and Duc Nguyen-Manh. April 21, 2020. Elucidating the differences in the molecular mechanism of receptor binding between 2019-nCoV and the SARS-CoV viruses using computational tools. bioRxiv preprint https://doi.org/10.1101/2020.04.21.053009

13 Shing Hei Zhan, Benjamin E. Deverman, Yujia Alina Chan3. May 2, 2020. SARS-CoV-2 is well adapted for humans. What does this mean for re-emergence? https://doi.org/10.1101/2020.05.01.073262 doi: bioRxiv preprint ${ }^{14}$ Sandrine Belouzard, Jean K. Millet, Beth N. Licitra and Gary R. Whittaker. Mechanisms of Coronavirus Cell Entry Mediated by the Viral Spike Protein. Viruses 2012, 4, 1011-1033; www.doi.org/10.3390/v4061011

${ }^{15}$ Xia, S., Liu, M., Wang, C. et al. Inhibition of SARS-CoV-2 (previously 2019-nCoV) infection by a highly potent pancoronavirus fusion inhibitor targeting its spike protein that harbors a high capacity to mediate membrane fusion. Cell Res 30, 343-355 (2020). https://doi.org/10.1038/s41422-020-0305-x

${ }^{16}$ P. V'kovski, M. Gultom, S. Steiner, J Kelly, J Russeil, ..., Ronald Dijkman. April 27, 2020. Disparate temperaturedependent virus - host dynamics for SARS-CoV-2 and SARS-CoV in the human respiratory epithelium. bioRxiv preprint doi: https://doi.org/10.1101/2020.04.27.062315.

17 S. Zhan, B. Deverman, Y. Chan. May 2, 2020. SARS-CoV-2 is well adapted for humans. What does this mean for reemergence? https://doi.org/10.1101/2020.05.01.073262

18 https://www.ncbi.nlm.nih.gov/nuccore/MN908947.1

19 https://trace.ncbi.nlm.nih.gov/Traces/sra/?run=SRR10168377 https://www.ncbi.nlm.nih.gov/sra/SRX6893154

20 https://www.ncbi.nlm.nih.gov/nuccore/MT121216

21 Liu P, Jiang J-Z, Wan X-F, Hua Y, Li L, Zhou J, et al. (2020) Are pangolins the intermediate host of the 2019 novel coronavirus (SARS-CoV-2)? PLoS Pathog 16(5): e1008421. https://doi.org/10.1371/journal.ppat.1008421

22 Luan J, Jin X, Lu Y, Zhang L. 30 March 2020. SARS - CoV - 2 spike protein favors ACE2 from Bovidae and Cricetidae. J Med Virol. 2020;1-8. https://doi.org/10.1002/jmv.25817

23 http://www.gromacs.org
} 